\title{
On Universality of Transition to Chaos Scenario in Nonlinear Systems of Ordinary Differential Equations of Shilnikov's Type
}

\author{
Maria Zaitseva1,2 \\ ${ }^{1}$ Scientific Research Institute of System Development, Russian Academy of Science, Moscow, Russia \\ ${ }^{2}$ Department of Computational Mathematics and Cybernetics, Moscow State University, Moscow, Russia \\ Email:mf.zaitseva@gmail.com
}

Received 16 March 2016; accepted 20 May 2016; published 23 May 2016

Copyright @ 2016 by author and Scientific Research Publishing Inc.

This work is licensed under the Creative Commons Attribution International License (CC BY).

http://creativecommons.org/licenses/by/4.0/

(c) (i) Open Access

\begin{abstract}
Several nonlinear three-dimensional systems of ordinary differential equations are studied analytically and numerically in this paper in accordance with universal bifurcation theory of Feigenbaum-Sharkovskii-Magnitsky [1] [2]. All systems are autonomous and dissipative and display chaotic behaviour. The analysis confirms that transition to chaos in such systems is performed through cascades of bifurcations of regular attractors.
\end{abstract}

\section{Keywords}

Nonlinear Differential Equations, Dynamical Chaos, Singular Attractor, FSM-Theory

\section{Introduction}

In recent years, many scientists around the world have tried to analyze and classify the variety of systems with chaotic behavior. In the article [3], Chen studied the problem of classification of quadratic autonomous dynamic systems of ordinary differential equations, in accordance with the extended Shilnikov's theorem. Systems of Shilnikov's type include a lot of well-known chaotic systems, such as the Lorenz system, Chua, Rssler, Chen systems. In that paper [3], the author classified systems of Shilnikov's type according to the type of chaotic attractors, existing in these systems: 1) chaos with homoclinic orbit; 2) chaos with heteroclinic orbit; 3) chaos of mixed type with homoclinic and heteroclinic orbits; 4) other types of chaos. The main aim of this work is to present the research of several model systems of ODEs of Shilnikov's type, mentioned in the above article, conducted in accordance with the trajectory approach of the universal bifurcation theory of FeigenbaumSharkovskii-Magnitskii (FSM) theory [1] [2]; the point is to confirm the existence of the only type of dynamic 
chaos and universal scenario of transition to chaos. This scenario begins with the Feigenbaum period doubling cascade of bifurcations of some original stable cycle, then continues with the Sharkovskii complete or incomplete subharmonic cascade of bifurcations of stable cycles of arbitrary period up to the cycle of period three and the Magnitskii complete or incomplete homoclinic cascade of bifurcations of stable cycles converging to homoclinic contours of equilibrium points or cycles.

\section{Results and Discussion}

In this work several chaotic systems from paper [3] are analysed. We use the fourth-order Runge-Kutta method to approximate the solutions of nonlinear autonomous systems of ODEs. Numerical simulations are prefaced by the research of system's equilibrium points, their type and stability.

\subsection{The Sprott(e) System}

The first system we study in this paper is a chaotic system Sprott(e) introduced by J. C. Sprott [4].

$$
\left\{\begin{array}{l}
\dot{x}=a y z, \\
\dot{y}=x^{2}-y, \\
\dot{z}=1-4 x,
\end{array}\right.
$$

where $a$ is a real parameter (Figure 1).

\subsubsection{Analytical Analysis}

The system (1) is dissipative everywhere in phase space since

$$
\operatorname{div} F(x, y, z)=-1<0 .
$$

Position of equilibrium points is defined by conditions:

$$
\left\{\begin{array}{l}
\text { ayz }=0 \\
x^{2}-y=0, \\
1-4 x=0
\end{array}\right.
$$

$x=0.25, y=0.0625, z=0$. Thus, the system (1) has one equilibrium point $O(0.25,0.0625,0)$. Let us find the roots of characteristic polynomial of Jacobi matrix in order to determine its type. We have the following form of linearization matrix of the right-hand side of the system (1):

$$
J=\left(\begin{array}{ccc}
0 & a z & a y \\
2 x & -1 & 0 \\
-4 & 0 & 0
\end{array}\right) .
$$

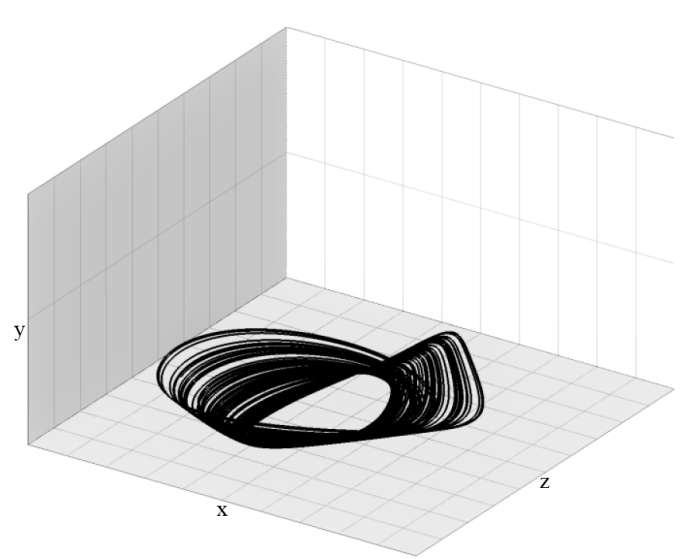

Figure 1. One of the irreglular attractors of the system for $a=1.01$. 
For $O(0.25,0.0625,0)$ it reads the form:

$$
J(O)=\left(\begin{array}{ccc}
0 & 0 & 0.0625 a \\
0.5 & -1 & 0 \\
-4 & 0 & 0
\end{array}\right)
$$

The characteristic equation for the Jacobi matrix at point $O$ looks as follows:

$$
\operatorname{det}(\lambda I-J(O))=\left|\begin{array}{ccc}
-\lambda & 0 & 0.0625 a \\
0.5 & -1-\lambda & 0 \\
-4 & 0 & -\lambda
\end{array}\right|=\lambda^{3}+\lambda^{2}+\frac{a}{4} \lambda+\frac{a}{4}=-\frac{1}{4}(\lambda+1)\left(a+4 \lambda^{2}\right) .
$$

Thus, we have $\lambda_{1}=-1, \quad \lambda_{2,3}= \pm \frac{i \sqrt{a}}{2}$ as roots of the characteristic equation, consequently our equilibrium point $O$ of the system (1) is a center.

\subsubsection{Numerical Simulations}

The cascade of bifurcations occurs with decreasing $a$. For $a \in[2.036,13.6]$ the phase trajectory of the system's solution appears as a stable cycle, at the value $a=2.035$ a period doubling bifurcation of the original stable cycle takes place and for $a \in[1.477,2.035]$ a double period cycle is observed, for $a \in[1.377,1.476]$ we found a cycle of period 4, for $a \in[1.355,1.376]$ we found a cycle of period 8, etc. The Feigenbaum attractor was found at $a=1.35$. Thus, in this system the Feigenbaum period doubling cascade of bifurcations can be observed (Figure 2).

Furthermore, at $a=1.2512$ a cycle of period 9 was found, for $a \in[1.2393,1.2399]$-a cycle of period 7 , for $a \in[1.2047,1.2077]$-a cycle of period 5 (Figure 3). That means, that the scenario of transition to chaos continues with the subharmonic cascade of bifurcations of stable cycles of arbitrary period in accordance with the Sharkovskii's order. For $a \in[1.1572,1.1875]$ a cycle of period 3 was found, its doubling for $a \in[1.1431,1.1571]$ and tripling for $a \in[1.1295,1.1301]$. That evidences the existence of the Feigenbaum cascade and complete subharmonic cascade for the cycle of period 3 (Figure 4). No cycles of homoclinic cascade of bifurcations were observed with further decreasing of the parameter $a$.
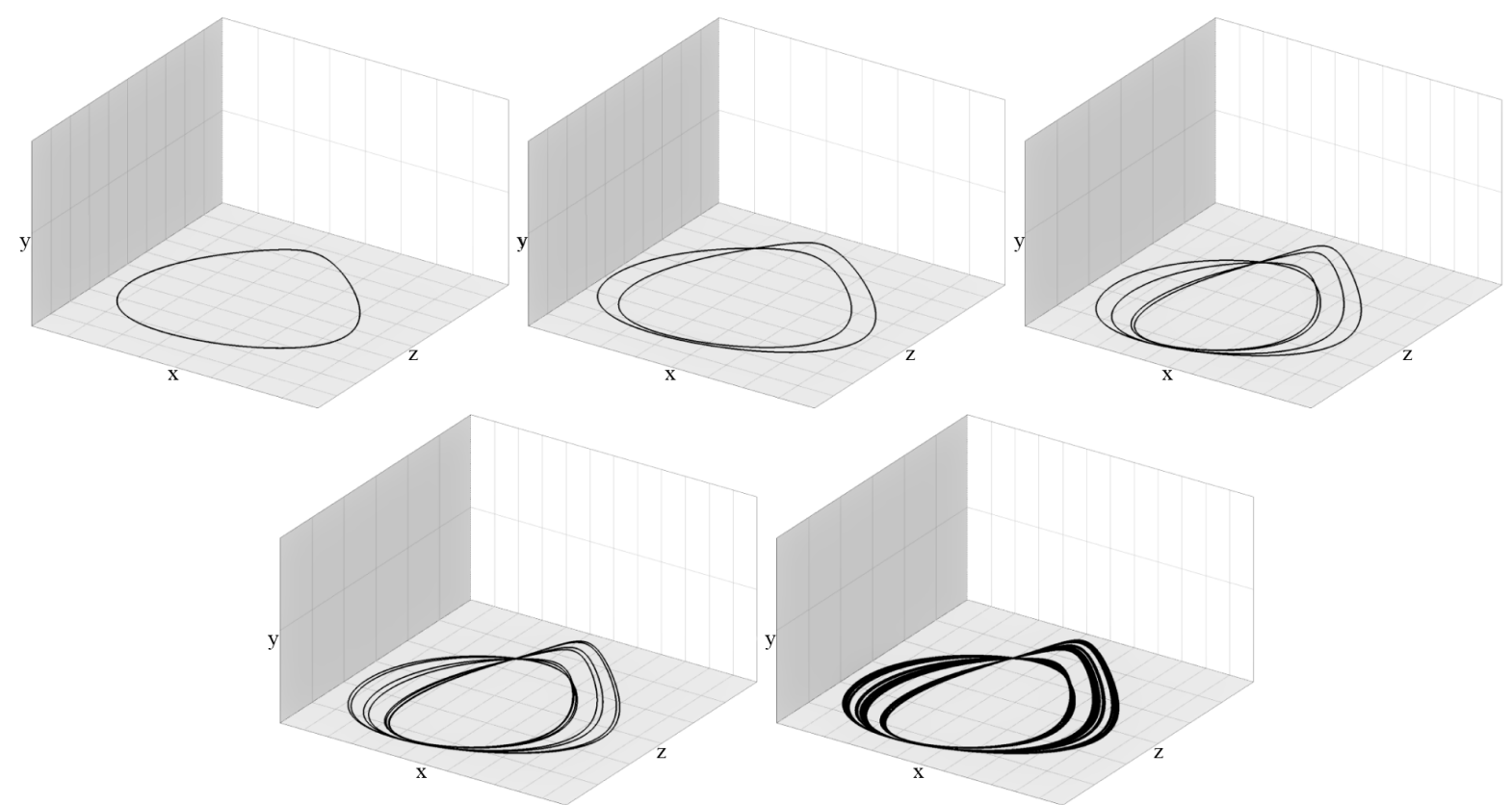

Figure 2. Cycles of the Feigenbaum cascade accordingly for $a=2.04, a=1.877, a=1.3826, a=1.3578$ and the Feigenbaum attractor for $a=1.35$. 

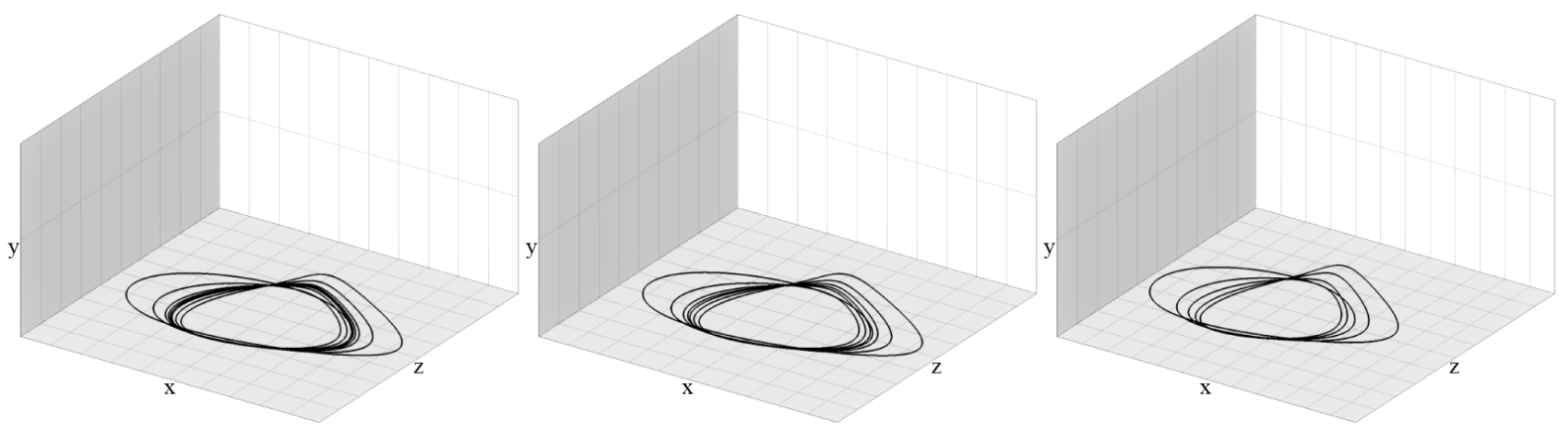

Figure 3. Cycles of periods 9, 7, 5 of the Sharkovskii subharmonic cascade for the parameter values $a=1.2512$, $a=1.2396, a=1.2067$.
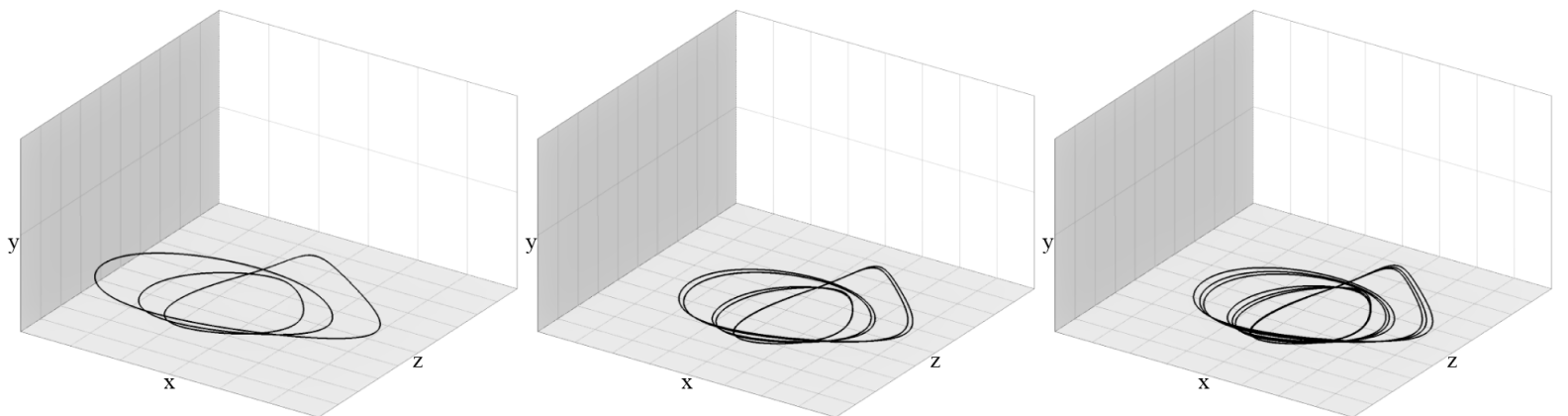

Figure 4. The cycle of period 3 for $a=1.1681$, its doubling for $a=1.1434$ and its tripling for $a=1.1299$.

\subsection{The Sprott(n) System}

We consider the other model system-Sprott (n) system [4].

$$
\left\{\begin{array}{l}
\dot{x}=a y, \\
\dot{y}=x+z^{2}, \\
\dot{z}=1+y-2 z,
\end{array}\right.
$$

where $a$ is a real parameter (Figure 5).

\subsubsection{Analytical Analysis}

Divergence of the system (7) is negative everywhere in phase space:

$$
\operatorname{div} F(x, y, z)=-2<0 .
$$

The system has one equilibrium point $O(-0.25,0,0.5)$.

The Jacobi matrix of this system at point $O$ is following:

$$
J(O)=\left(\begin{array}{ccc}
0 & a & 0 \\
1 & 0 & 1 \\
0 & 1 & -2
\end{array}\right)
$$

We shall find the eigenvalues of $J(O)$ from the characteristic equation

$$
\operatorname{det}(\lambda I-J(O))=\left|\begin{array}{ccc}
-\lambda & a & 0 \\
1 & -\lambda & 1 \\
0 & 1 & -2-\lambda
\end{array}\right|=\lambda^{3}+2 \lambda^{2}-(1+a) \lambda-2 a=0 .
$$




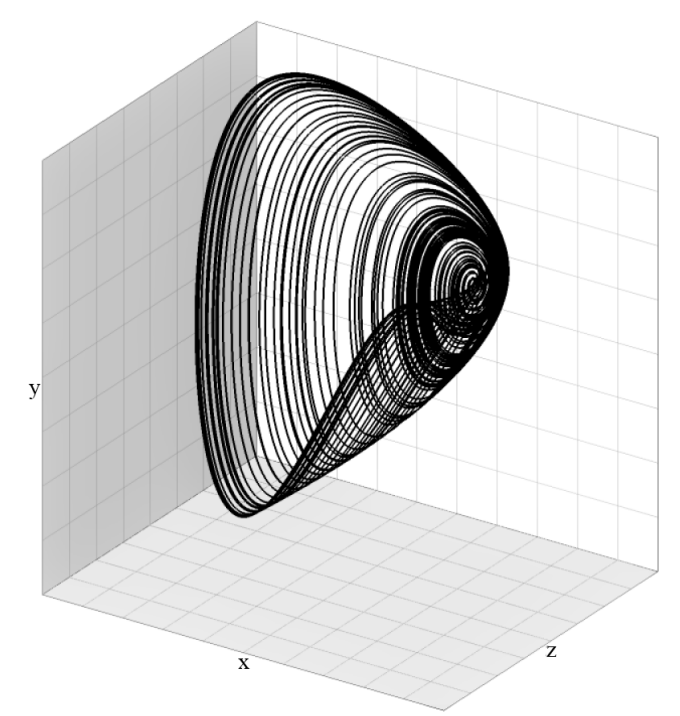

Figure 5. One of the chaotic attractors for $a=-1.688$.

Let us take $\lambda=\mu-\frac{2}{3}$. We have:

$$
\mu^{3}+p \mu+q=0
$$

where $p=-a-\frac{7}{3}, q=-\frac{4 a}{3}+\frac{34}{27}$.

The equation (11) has one real and two complex conjugate roots if the discriminant $\Delta>0$. The discriminant (11) is determined by

$$
\Delta=\left(\frac{q}{2}\right)^{2}+\left(\frac{p}{3}\right)^{3}
$$

We have:

$$
\Delta=\frac{1}{27}\left(-a^{3}+5 a^{2}-39 a-2\right)>0 .
$$

Thus, when (13) holds, the original characteristic equation (10) also has one real and two complex conjugate roots.

An equilibrium point is a saddle-focus if the real root is negative and the real part of complex conjugate roots is positive. Let us determine these conditions.

Notice that $\lambda_{i}=\mu_{i}-\frac{2}{3}$, where $1 \leq i \leq 3$ are the roots of (10). Consequently, the inequality $\lambda_{1}<0$ reads as $\alpha+\beta<\frac{2}{3}, \operatorname{Re}\left(\lambda_{2,3}\right)>0$ reads as $\alpha+\beta<-\frac{4}{3}$.

We have:

$$
\sqrt[3]{-\frac{q}{2}+\sqrt{\Delta}}+\sqrt[3]{-\frac{q}{2}-\sqrt{\Delta}}<-\frac{4}{3}
$$

Thus, we get that under the condition (13) for the existence of one real and two complex conjugate roots and the condition (14) for negativity of a real root and the positivity of complex conjugate roots, the equilibrium point $O(-0.25,0,0.5)$ is an unstable saddle-focus.

\subsubsection{Numerical Simulations}

For values of the bifurcation parameter $a<-2.68$ there is a stable cycle in the system. At $a=-2.67$ the 
Feigenbaum cascade begins with birth of a double period cycle which exists for $a \in[-2.67,-2.26]$, then a cycle of period 4 for $a \in[-2.25,-2.17]$, a cycle of period 8 for $a \in[-2.16,-2.13518]$ emerge. At $a=-2.131$ the period doubling cascade comes to the end with the formation of a Feigenbaum attractor (Figure 6).

Then the scenario of transition to chaos continues with the subharmonic cascade of bifurcations. We found the following cycles: for $a \in[-2.05865,-2.05864]$ a cycle of period 9, for $a \in[-2.05004,-2.04972]$ a cycle of period 7, for $a \in[-2.0268,-2.02464]$ a cycle of period 5, for $a \in[-1.96535,-1.95324]$ a cycle of period 3 (Figure 7). We also found doubling of period 3 cycle for $a \in[-1.95323,-1.94758]$ and its tripling for $a=[-1.94232,-1.94212]$, confirming the presence of the complete subharmonic cascade for a cycle of period 3 (Figure 8). For $a \in[-1.86125,-1.85959]$ a homoclinic cycle of period 4 was found (Figure 9). This implies that the scenario further continues with Magnitskii homoclinic cascade of bifurcations of stable cycle with further increase of the parameter $a$.

\subsection{The Burke-Shaw System}

The last system we study in this paper is the Burke-Shaw system derived by B. Burke and R. Shaw from the Lorenz system [5].

$$
\left\{\begin{array}{l}
\dot{x}=-a(x+y), \\
\dot{y}=-y-a x z, \\
\dot{z}=a x y+b,
\end{array}\right.
$$

where $a$ and $b$ are real parameters (Figure 10).

\subsubsection{Analytical Analysis}

Divergence of the system (15) is negative in the area
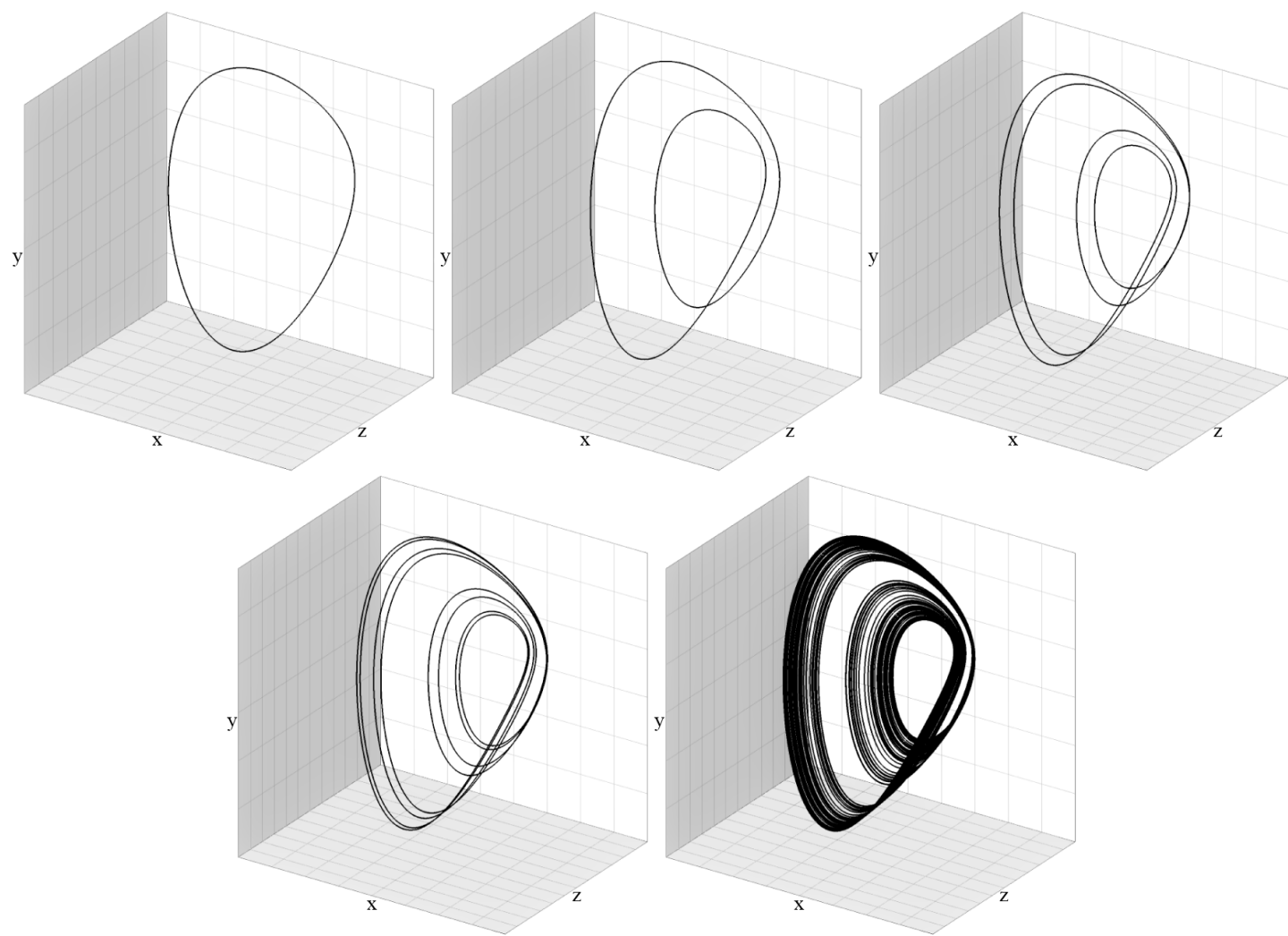

Figure 6. Cycles of period doubling cascade of bifurcations accordingly for the parameter values $a=-2.91, a=-2.39$, $a=-2.18, \quad a=-2.13522, \quad a=-2.131$. 

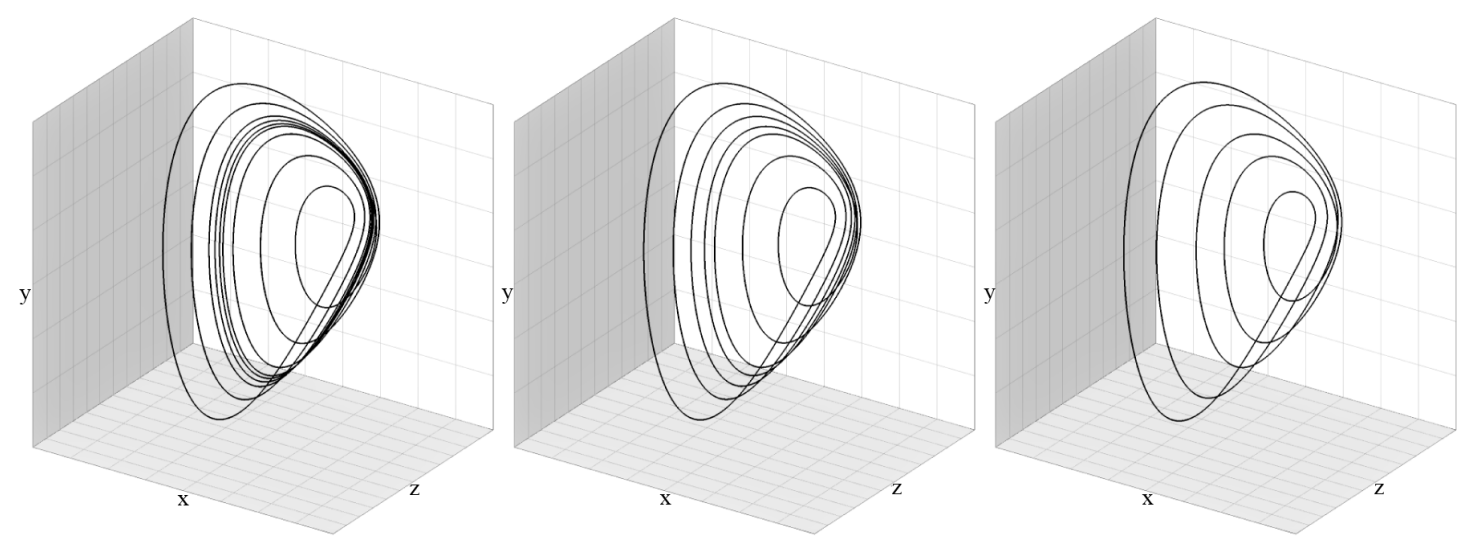

Figure 7. Cycles of the periods 9, 7, 5 for $a=-2.05865, a=-2.04985, a=-2.02658$ correspondingly.
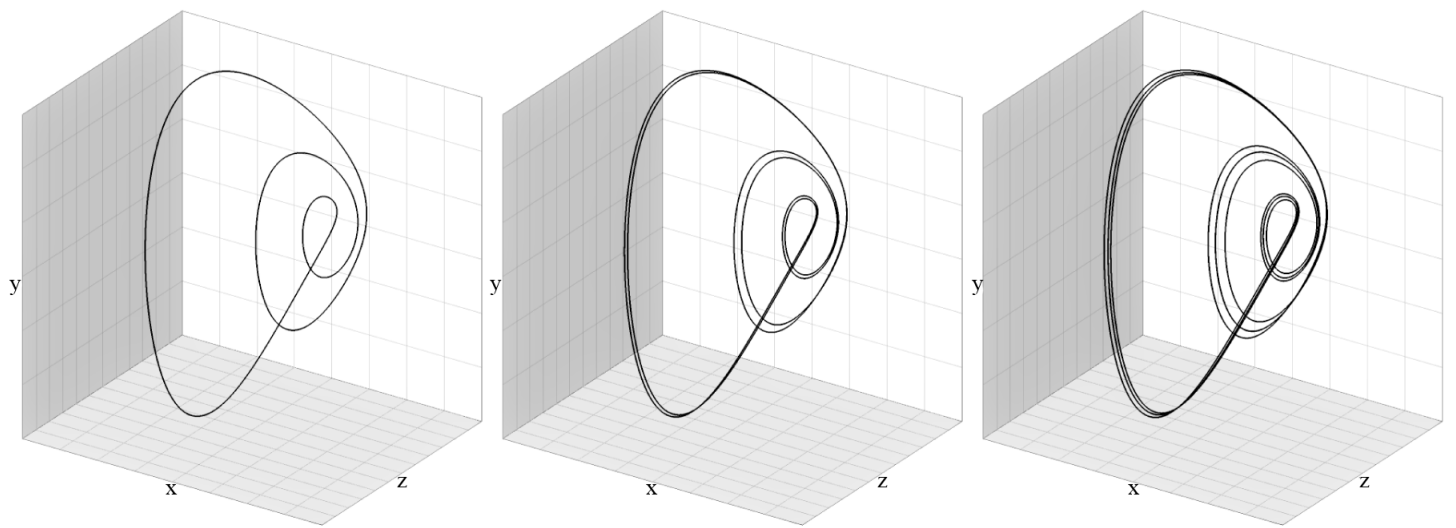

Figure 8. The cycle of period 3 and its doubling and tripling for $a=-1.95829, a=-1.94764$ and $a=-1.94213$ in corresponding order.
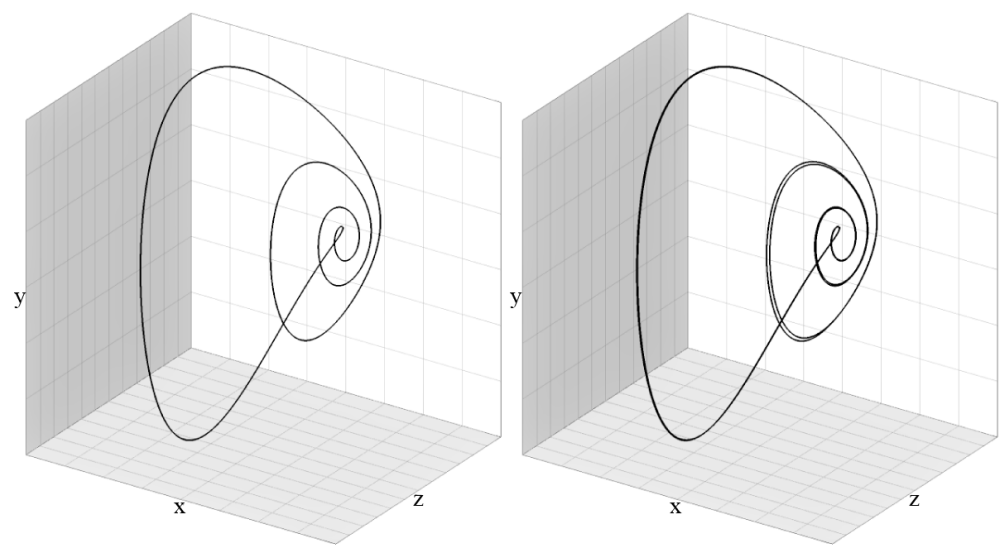

Figure 9. The homoclinic cycle of period 4 for $a=-1.86076$ and its doubling for $a=-1.85888$.

$$
a>-1
$$

Taking into consideration the condition (16) we have $x= \pm \sqrt{\frac{b}{a}}, y=\mp \sqrt{\frac{b}{a}}, \quad z=\frac{1}{a}$. The system has two equilibrium states $O_{1}\left(\sqrt{\frac{b}{a}},-\sqrt{\frac{b}{a}}, \frac{1}{a}\right)$ and $O_{2}\left(-\sqrt{\frac{b}{a}}, \sqrt{\frac{b}{a}}, \frac{1}{a}\right)$, which are symmetric with respect to the $z$ axis. 


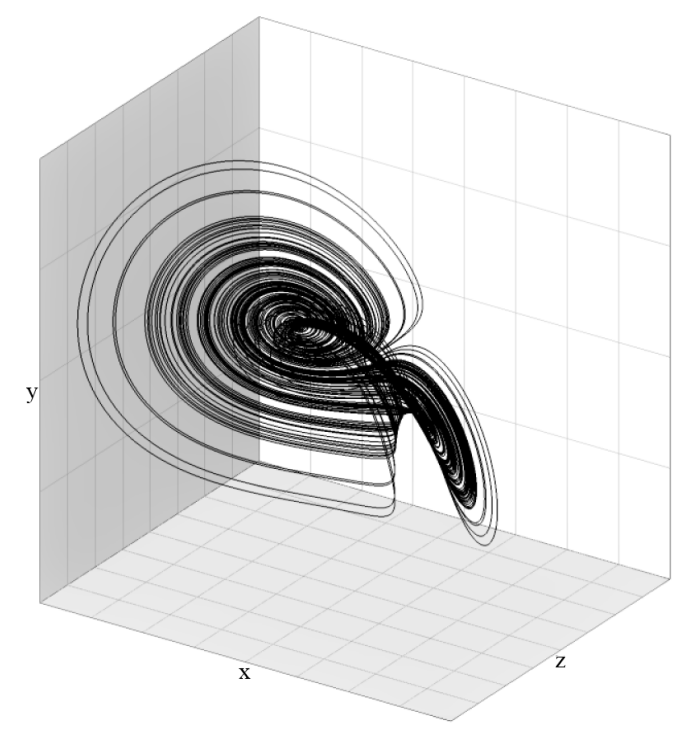

Figure 10. The chaotic attractor for $b=13.5, \quad a=10.0$.

Eigenvalues of a matrix of linearization are defined at these points by the characteristic equation:

$$
\lambda^{3}+(a+1) \lambda^{2}+a b \lambda+2 a^{2} b=0 .
$$

Furthermore, we shall notice that if

$$
a>-1,
$$

then

$$
\Delta=\frac{1}{108} a^{2} b\left(8 a^{3}+a^{2}(71 b+24)+a\left(4 b^{2}-38 b+24\right)-b+8\right)>0
$$

and

$$
\sqrt[3]{-\frac{q}{2}+\sqrt{\Delta}}+\sqrt[3]{-\frac{q}{2}-\sqrt{\Delta}}<-\frac{2(a+1)}{3}
$$

The characteristic polynomials of linearization matrix at the equilibrium points $O_{1}\left(\sqrt{\frac{b}{a}},-\sqrt{\frac{b}{a}}, \frac{1}{a}\right)$ and $O_{2}\left(-\sqrt{\frac{b}{a}}, \sqrt{\frac{b}{a}}, \frac{1}{a}\right)$ each have one negative real root and a pair of complex conjugate roots with positive real part, therefore, under these conditions the equilibrium points of the system are unstable saddle-focuses.

\subsubsection{Numerical Simutions}

We found that shift of dynamics of the system towards more complex solutions (which takes place with the growth of the parameter $b$ ) is in agreement with the FSM-scenario. We fix the value of the parameter $a=10.0$.

For $b \in[2.392,3.451]$ a stable cycle is the only attractor of the system. Next the Feigenbaum period doubling cascade of the original cycle starts: for $b \in[3.452,3.591]$ a double period cycle, for $b \in[3.592,3.622]$ a cycle of period 4 , at $b=3.6287$ the Feigenbaum attractor (Figure 11).

Several cycles from Sharkovskii cascade were observed: a cycle of period 9 for $b \in[3.8307,3.8308]$, a cycle of period $7 b \in[3.8561,3.8576]$, a cycle of period 5 for $b \in[3.9158,3.9224]$, a cycle of period 3 for $b \in[4.052,4.074]$ (Figure 12).

With further increase of the bifurcation parameter we found a cycle of the homoclinic cascade with 4 loops at 


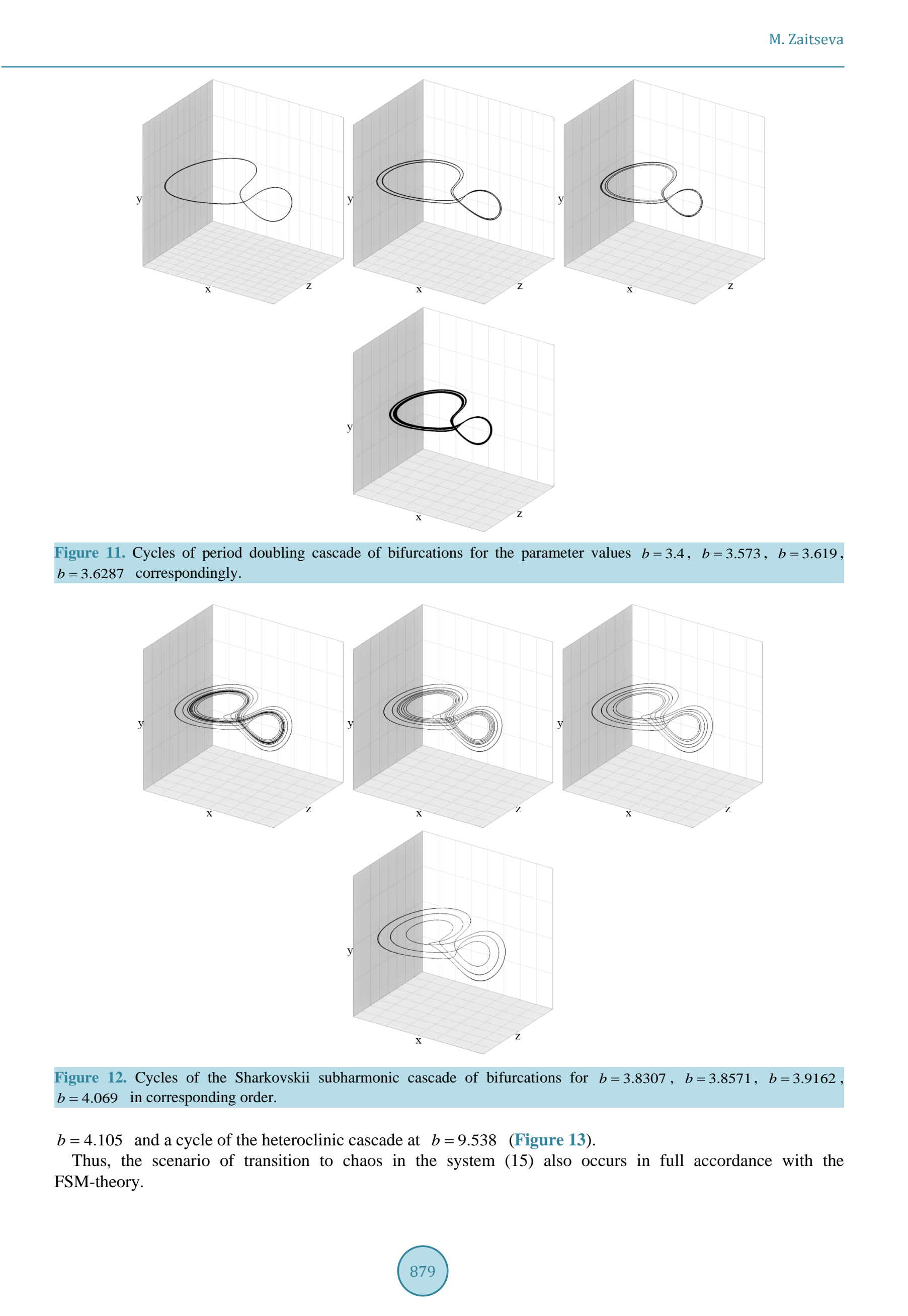




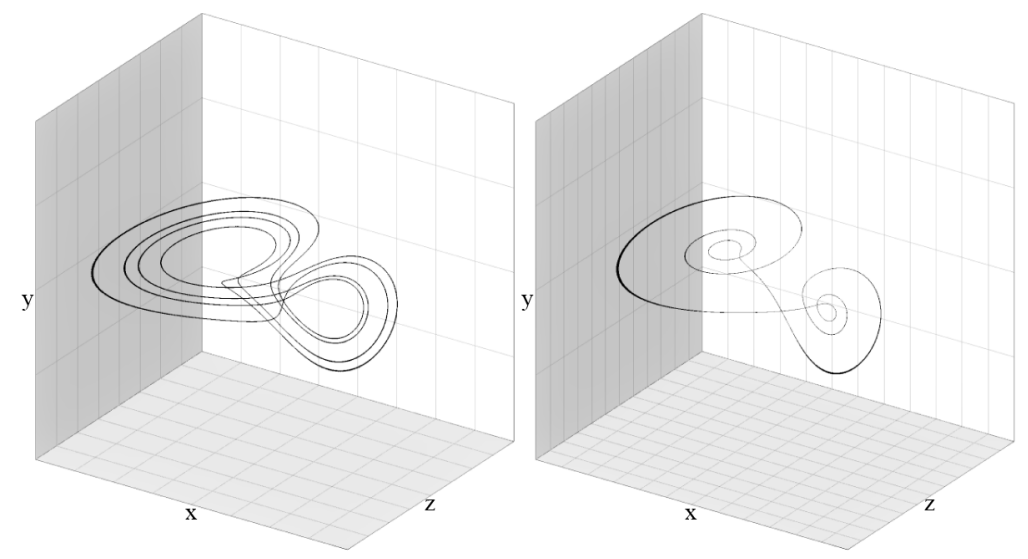

Figure 13. The homoclinic and heteroclinic cycles.

\section{Conclusion}

The present paper carries out analytical and numerical study of three chaotic systems of Shilnikov's type referred in Chen's article [3], two Sprott's systems-the Sprott(e) and the Sprott(n) and the Burke-Shaw system. For these systems, bifurcation parameters and types of fixed points were found, as well as the occurrence of initial cycles of the bifurcation cascades and the occurrence of chaotic attractors. With variation of the parameters, we studied phase portraits in this three systems and revealed the same scenario of singular attractors occurrence, which fully corresponded to the mechanism of transition to chaos by Feigenbaum-Sharkovskii-Magnitskii. So, despite classification [3], all systems seem to fall into one category according to the FSM theory, as chaotic attractors have the same nature.

\section{Acknowledgements}

The author is grateful to Prof. N.A. Magnitskii for critical comments concerning analysis of results presented in this paper, to O.I. Ryabkov and D.A. Burov for valuable discussions.

\section{References}

[1] Magnitskii, N.A. and Sidorov, S.V. (2006) New Methods for Chaotic Dynamics. World Scientific, Singapore.

[2] Magnitskii, N.A. (2008) Universal Theory of Dynamical Chaos in Nonlinear Dissipative Systems of Differential Equations. Communications in Nonlinear Science and Numerical Simulation, 13, 416-433. http://dx.doi.org/10.1016/j.cnsns.2006.05.006

[3] Chen, B. (2013) The Related Extension and Application of the Shilnikov Theorem. Journal of Applied Mathematics, 2013, Article ID: 287123. http://dx.doi.org/10.1155/2013/287123

[4] Sprott, J.C. (1994) Some Simple Chaotic Flows. Physical Review E, 50, R647.

[5] Shaw, R. (1981) Strange Attractors, Chaotic Behavior and Information Flow. Zeitschrift für Naturforschung A, 36, 80-112. http://dx.doi.org/10.1515/zna-1981-0115 